\title{
Efficacy of transcutaneous cardiac pacing for transient asystole caused by trigeminocardiac reflex: illustrative case
}

\author{
Shuhei Yamada, MD, ${ }^{1,2}$ Yoshihiro Yano, MD, ${ }^{1}$ Toshiaki Fujita, MD, PhD, ${ }^{1}$ and Mamoru Taneda, MD, PhD ${ }^{1}$ \\ ${ }^{1}$ Department of Neurosurgery, Hanwa Memorial Hospital, Osaka, Japan; and ${ }^{2}$ Department of Neurosurgery, Graduate School of Medicine, Osaka University, Osaka, Japan
}

BACKGROUND Trigeminocardiac reflex (TCR) is a brainstem reflex caused by stimulation of the trigeminal nerve, which results in bradycardia, hypotension, and asystole. TCR can occur during any neurosurgical procedure. Initially, it is managed via the immediate removal of the stimulus from the trigeminal nerve. If asystole persists after intravenous atropine or glycopyrrolate, chest compression or transcutaneous cardiac pacing may be considered. The authors present the first case of TCR that was successfully managed with transcutaneous cardiac pacing.

OBSERVATIONS A 51-year-old man presented with aneurysmal subarachnoid hemorrhage. Although he had no history of cardiac disease and there were no abnormal findings on electrocardiography, transient asystole due to TCR occurred during craniotomy. The patient's heart rate spontaneously recovered after the immediate discontinuation of the procedure. The authors completed aneurysm clipping with transcutaneous cardiac pacing because intravenous atropine was not effective in preventing TCR. There were no complications associated with intraoperative asystole or transcutaneous cardiac pacing, and the patient was discharged without neurological deficits.

LESSONS TCR can be appropriately managed with the immediate discontinuation of intraoperative procedures. Furthermore, transcutaneous cardiac pacing may be considered for persistent TCR with poor response to intravenous atropine or glycopyrrolate.

https://thejns.org/doi/abs/10.3171/CASE21198

KEYWORDS transcutaneous cardiac pacing; trigeminocardiac reflex; transient asystole; cerebral aneurysm; clipping

Trigeminocardiac reflex (TCR) is a brainstem reflex caused by stimulation of the trigeminal nerve, which results in bradycardia, hypotension, and asystole. ${ }^{1}$ TCR may occur during not only ocular, craniofacial, and skull base surgeries ${ }^{2}$ but also neurointerventional treatments ${ }^{3}$ and supratentorial cerebral aneurysm surgeries. ${ }^{4-6}$ Initially, it is treated via immediate removal of the stimulus from the trigeminal nerve. ${ }^{2}$ Next, if bradycardia and hypotension persist, intravenous atropine or glycopyrrolate should be administered. ${ }^{3}$ Moreover, if asystole persists, chest compression and transcutaneous cardiac pacing may be considered. ${ }^{7}$ Herein, we present the first case of TCR occurring during emergency surgery for a ruptured cerebral aneurysm that was successfully managed with transcutaneous cardiac pacing.

\section{Illustrative Case}

A 51-year-old man presented to our emergency department due to sudden-onset headache. Computed tomography (CT) and CT angiography revealed subarachnoid hemorrhage (Fig. 1A; World
Federation of Neurosurgical Societies grade 1, Fisher group 3) due to a ruptured anterior communicating artery aneurysm (Fig. 1B; largest diameter, $7 \mathrm{~mm}$ ). Moreover, he had no history of cardiac disease, and there were no abnormal findings on electrocardiography upon admission (Fig. 2).

On the second day of hospitalization, the patient underwent right frontotemporal (pterional) craniotomy under general anesthesia. For preinduction, he received dexmedetomidine, and anesthesia was maintained with remifentanil, propofol, and rocuronium. Transient asystole lasting for a few seconds occurred when the dura was detached from the skull. However, the patient's heart rate spontaneously recovered after immediate discontinuation of the procedure. Despite provision of a bolus infusion of atropine sulfate and infiltration anesthesia of the dura with $1 \%$ lidocaine, TCR still occurred after dural manipulation. Therefore, craniotomy was performed with transcutaneous cardiac pacing (Fig. 3A). Because mechanical

ABBREVIATIONS CPA = cerebellopontine angle; $C T$ = computed tomography; ICA = internal carotid artery; TCR = trigeminocardiac reflex. INCLUDE WHEN CITING Published August 16, 2021; DOI: 10.3171/CASE21198.

SUBMITTED April 7, 2021. ACCEPTED April 18, 2021.

(C) 2021 The authors, CC BY-NC-ND 4.0 (http://creativecommons.org/licenses/by-nc-nd/4.0/). 

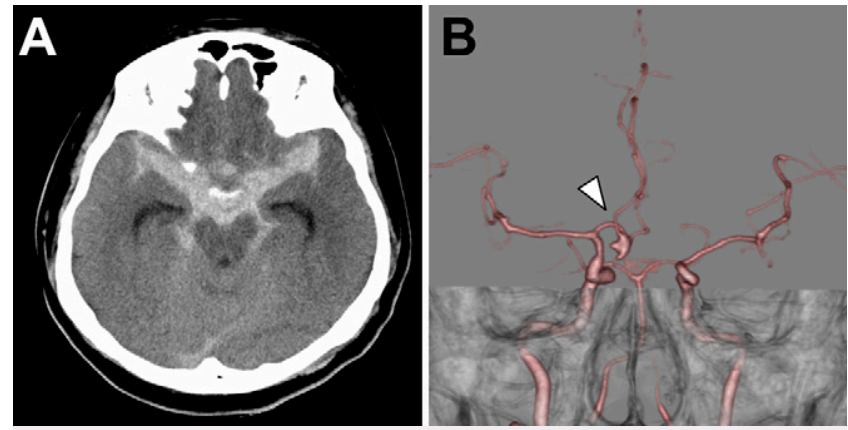

FIG. 1. CT scan upon admission revealed subarachnoid hemorrhage (A). Preoperative three-dimensional CT angiography showed a 7-mm saccular aneurysm in the anterior communicating artery ( $\mathbf{B}$, arrowhead).

stimulation of the internal carotid artery (ICA) also decreased the patient's heart rate and activated transcutaneous cardiac pacing (Fig. $3 \mathrm{~B})$, utmost caution was taken during surgery. The aneurysm could be successfully clipped with a straight Sugita II mini clip (Fig. 3C; no. 100, $8 \mathrm{~mm}$ ). No changes in pulse rate or blood pressure were observed during the subsequent procedures, including dural closure.

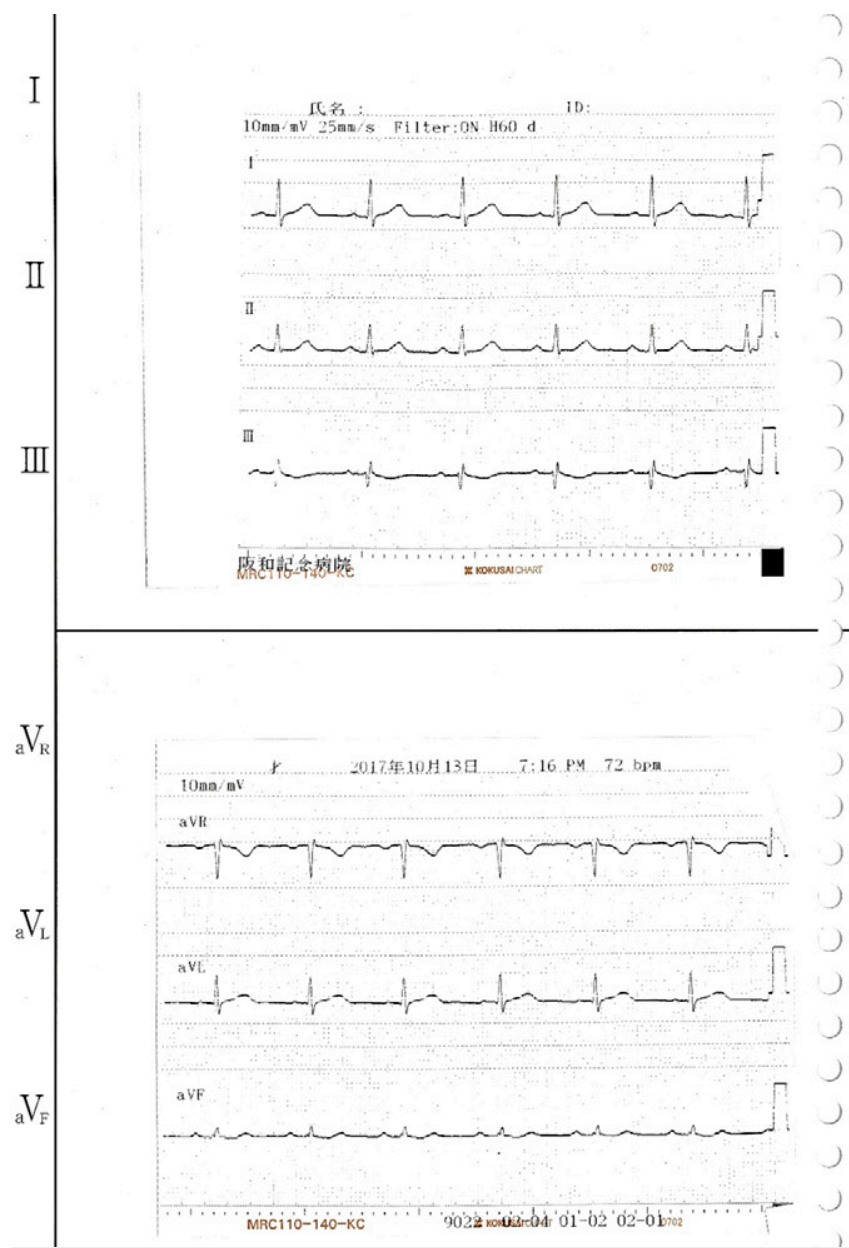

FIG. 2. Electrocardiography upon admission showed no abnormal findings.
There were no perioperative complications associated with intraoperative asystole or transcutaneous cardiac pacing. The postoperative period was uneventful. The patient was discharged without neurological deficits on day 21. No aneurysm recurrence or neurological sequelae were observed during 4 years of follow-up (modified Rankin scale score of 0 ).

\section{Discussion}

\section{Observations}

TCR is a brainstem reflex caused by stimulation of the trigeminal nerve. ${ }^{1}$ The term "TCR" was introduced in $1988 .{ }^{8}$ Moreover, in 1999, TCR was defined as hypotension with a mean arterial blood pressure decrease $(>20 \%)$ and bradycardia $\left(<60\right.$ beats/minute). ${ }^{9}$ The oculocardiac reflex, a subtype of TCR, ${ }^{1,8}$ occurs during ocular surgery, with an incidence rate of $90 \%{ }^{2}$ About $8 \%-18 \%$ and $11 \%$ of patients who undergo skull base ${ }^{2}$ and cerebellopontine angle (CPA) surgeries, ${ }^{9}$ respectively, experience TCR.

Transient asystole attributed to TCR is not rare. That is, it is observed in $2.4 \%$ and $2.3 \%$ of patients who undergo $\mathrm{CPA}^{9}$ and supratentorial cerebral aneurysm surgeries, respectively. ${ }^{6}$ Furthermore, some studies have reported transient asystole due to TCR during not only endoscopic transsphenoidal surgery ${ }^{10,11}$ and endovascular treatments ${ }^{3,12}$ but also

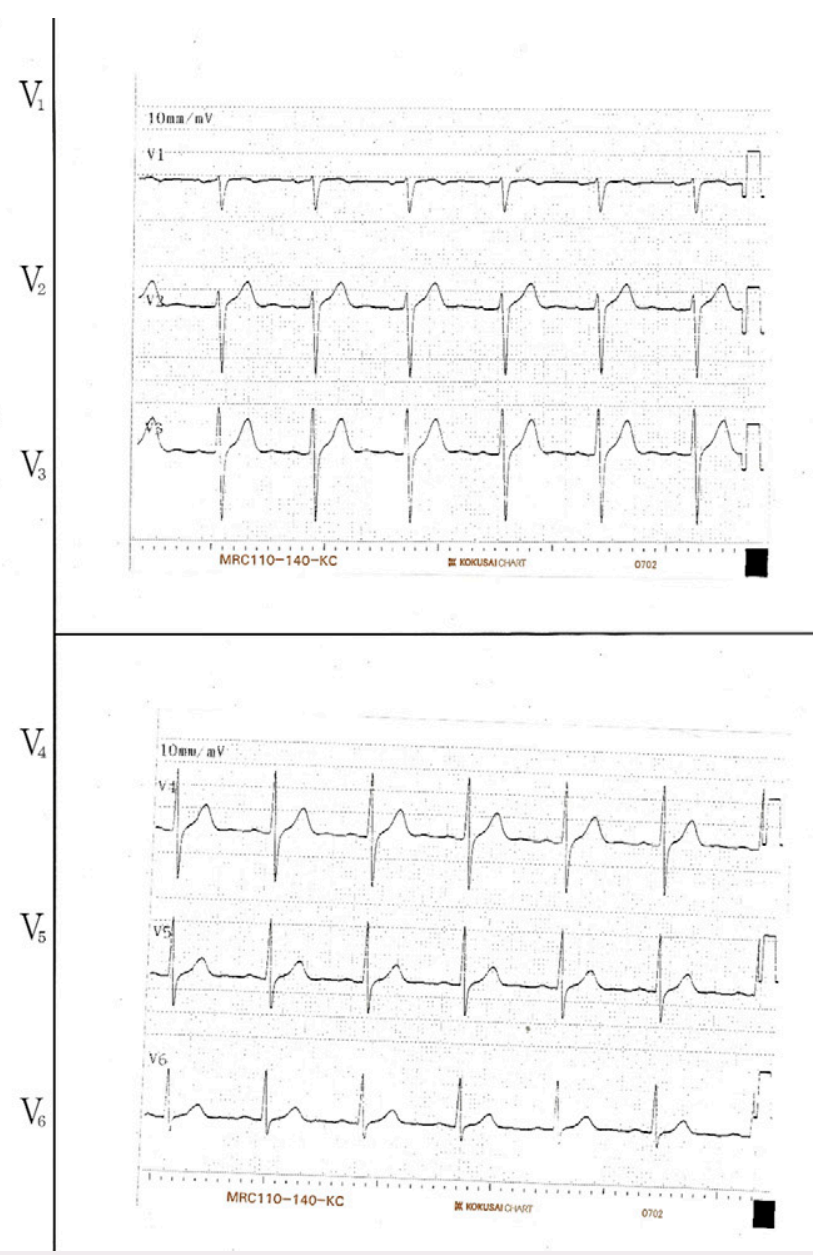



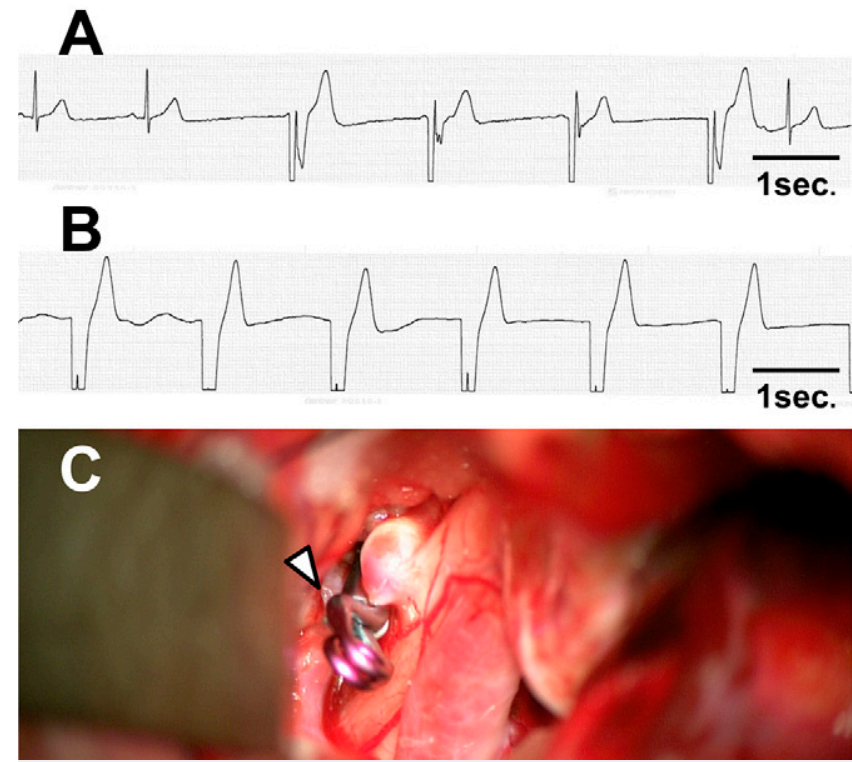

FIG. 3. Intraoperative electrocardiography showed the activation of transcutaneous cardiac pacing for transient asystole during craniotomy (A) and traction of the ICA (B). The aneurysm could be successfully clipped with a straight Sugita II mini clip (C, arrowhead).

skin flap elevation. ${ }^{13}$ Therefore, the risk of TCR should always be considered by all neurosurgeons or anesthesiologists.

When TCR develops, the stimulus must be removed from the trigeminal nerve immediately. ${ }^{2}$ If bradycardia and hypotension are persistent, intravenous atropine or glycopyrrolate should be administered. ${ }^{3}$ Moreover, local anesthetic infiltration may be effective. ${ }^{14}$ If asystole or atropine-resistant bradycardia is persistent, chest compression and transcutaneous cardiac pacing can be considered. ${ }^{7}$ Furthermore, alternative treatment modalities such as endovascular treatment instead of craniotomy should be considered. The outcome of endovascular coiling for ruptured cerebral aneurysms is not inferior to that of neurosurgical clipping. ${ }^{15,16}$ Thus, we considered switching the procedure to coil embolization in this case. However, transcutaneous cardiac pacing, in addition to the administration of intravenous atropine, infiltration anesthesia with lidocaine, and minimal mechanical stimulation of the ICA, facilitated complete neurosurgical clipping. On the basis of our experience, the vibration caused by intraoperative pacing must not be disregarded in microsurgery, because it poses some risks.

Factors correlated with TCR, such as hypoxemia, hypercapnia, light general anesthesia, and use of $\beta$-blockers, calcium channel blockers, and remifentanil, $2,9,14,17$ should be eliminated if possible. However, in this case, the only associated factor was the use of remifentanil, which is widely used in neurosurgical anesthesia because of its rapid metabolism. ${ }^{18}$ Thus, its risks and benefits may require cautious consideration.

Most defibrillators in the operating room have a transcutaneous cardiac pacing function. Pacing pads can be easily applied even when the patient is under general anesthesia, ${ }^{19}$ and transcutaneous cardiac pacing can be an option for atropine-resistant bradycardia in the emergency department. ${ }^{20}$ The authors of a previous report used transcutaneous cardiac pacing as a prophylactic for TCR occurring during sphenopalatine ganglion implantation. ${ }^{7}$ However, to the best of our knowledge, there is no report about TCR occurring during neurosurgery that was successfully managed with intraoperative transcutaneous cardiac pacing. Hence, further studies must be conducted to validate our results.

\section{Lessons}

TCR can occur in any neurosurgical procedure. The most appropriate treatment for TCR is the immediate discontinuation of intraoperative procedures. Furthermore, transcutaneous cardiac pacing may be an option for persistent TCR with poor response to intravenous atropine or glycopyrrolate.

\section{Acknowledgments}

We thank Enago for the English-language review.

\section{References}

1. Meuwly C, Golanov E, Chowdhury T, Erne P, Schaller B. Trigeminal cardiac reflex: new thinking model about the definition based on a literature review. Medicine (Baltimore). 2015;94(5):e484.

2. Schaller B, Cornelius JF, Prabhakar $\mathrm{H}$, et al. The trigemino-cardiac reflex: an update of the current knowledge. J Neurosurg Anesthesiol. 2009;21(3):187-195.

3. Chowdhury T, Rizk AA, Azazi EA, Venkatraghavan L, Shankar JS, Schaller B. Brain and heart crosstalk during neurointerventional procedures: the role of the trigeminocardiac reflex: an updated systematic review. J Neurosurg Anesthesiol. Published online August 28, 2020. doi: https://doi.org/10.1097/ANA.0000000000000723.

4. Spiriev T, Kondoff S, Schaller B. Trigeminocardiac reflex during temporary clipping in aneurismal surgery: first description. J Neurosurg Anesthesiol. 2011;23(3):271-272.

5. Kitabayashi M, Nakamura K, Murata T. A case of trigeminocardiac reflex in the pterional approach. Article in Japanese. No Shinkei Geka. 2012;40(10):903-907.

6. Shibata T, Sakai N, Tani S, et al. Transient asystole during surgery for intracranial unruptured aneurysms caused by excitation of the trigemino-vagal reflex: two case reports. Jpn J Neurosurg. 2014;23(8):673-677.

7. Hammad Y, Mootz A, Klein K, Zuniga JR. Trigeminocardiac reflex induced by maxillary nerve stimulation during sphenopalatine ganglion implantation: a case series. Brain Sci. 2020;10(12):973.

8. Shelly MP, Church JJ. Bradycardia and facial surgery [letter]. Anaesthesia. 1988:43(5):422.

9. Schaller B, Probst R, Strebel S, Gratzl O. Trigeminocardiac reflex during surgery in the cerebellopontine angle. J Neurosurg. 1999;90(2):215-220.

10. Onodera $Y$, Takaoka S, Oda S, et al. Cardiac arrest during endoscopic transsphenoidal pituitary surgery. J Japan Soc Clin Anesth. 2012;32(5):795-797.

11. Schaller B. Trigemino-cardiac reflex during transsphenoidal surgery for pituitary adenomas. Clin Neurol Neurosurg. 2005;107(6):468-474.

12. Crockett MT, Robinson AE, Aneja H, Phillips TJ. Posterior meningeal artery DMSO injection resulting in reproducible asystole prior to Onyx therapy of a dural arteriovenous fistula: a previously undescribed variant of the trigeminocardiac reflex or a new phenomenon? BMJ Case Rep. 2017;2017:bcr2017221033.

13. Prabhakar H, Rath GP, Arora R. Sudden cardiac standstill during skin flap elevation in a patient undergoing craniotomy. J Neurosurg Anesthesiol. 2007;19(3):203-204.

14. Lang S, Lanigan DT, van der Wal M. Trigeminocardiac reflexes: maxillary and mandibular variants of the oculocardiac reflex. Can J Anaesth. 1991;38(6):757-760.

15. Molyneux A, Kerr R, Stratton I, et al. International Subarachnoid Aneurysm Trial (ISAT) of neurosurgical clipping versus endovascular coiling in 2143 patients with ruptured intracranial aneurysms: a randomised trial. Lancet. 2002;360(9342):1267-1274. 
16. Spetzler RF, McDougall CG, Zabramski JM, et al. Ten-year analysis of saccular aneurysms in the Barrow Ruptured Aneurysm Trial. J Neurosurg. 2019;132(3):771-776.

17. Usami K, Kamada K, Kunii N, Tsujihara H, Yamada Y, Saito N. Transient asystole during surgery for posterior fossa meningioma caused by activation of the trigeminocardiac reflex: three case reports. Neurol Med Chir (Tokyo). 2010;50(4):339-342.

18. Del Gaudio A, Ciritella P, Perrotta F, et al. Remifentanil vs fentanyl with a target controlled propofol infusion in patients undergoing craniotomy for supratentorial lesions. Minerva Anestesiol. 2006;72(5):309-319.

19. Sovari AA. Transcutaneous cardiac pacing technique. Medscape. Updated December 13, 2020. Accessed March 2, 2021. https:// emedicine.medscape.com/article/98939-technique.

20. Bektas F, Soyuncu S. The efficacy of transcutaneous cardiac pacing in ED. Am J Emerg Med. 2016;34(11):2090-2093.

\section{Disclosures}

The authors report no conflict of interest concerning the materials or methods used in this study or the findings specified in this paper.

\section{Author Contributions}

Conception and design: Yamada, Fujita. Acquisition of data:

Yamada, Fujita. Analysis and interpretation of data: Yamada, Fujita. Drafting the article: Yamada. Critically revising the article: Yamada. Reviewed submitted version of manuscript: Yamada. Approved the final version of the manuscript on behalf of all authors: Yamada. Administrative/technical/material support: Yamada, Yano. Study supervision: Yamada, Taneda.

\section{Correspondence}

Shuhei Yamada: Hanwa Memorial Hospital, Osaka, Japan. s-yamada@ nsurg.med.osaka-u.ac.jp. 